\title{
Test Results of the Third LHC Main Quadrupole Magnet Prototype at CEA/Saclay
}

\author{
F. Simon, C. Gourdin, T. Schild, J. Deregel, A. Devred, B. Hervieu, M. Peyrot, J. M. Rifflet, T. Tortschanoff, \\ T. Ogitsu, and K. Tsuchiya
}

\begin{abstract}
The construction of the third second-generation main quadrupole magnet prototype for LHC has been completed at CEA/Saclay in November 2000.

The magnet was tested at $1.9 \mathrm{~K}$. Similarly to the two first ones, this prototype has exceeded the operating current in one training step and exhibited excellent training memory after a thermal cycle. This paper describes the quench performance and quench start localization determined by means of voltage-taps and a quench antenna system developed in collaboration with KEK.

As this magnet was equipped with capacitive gauges, the stresses during cool-down and powering have been recorded and are in agreement with FE computations. The newly designed quench heaters have improved efficiency and reproducibility compared to those of the first generation.

Magnetic measurements have been performed at various stages. The cold measurements show minor differences with those at room temperature and are similar to those of the two first magnets of this design.

These results prove that the magnets are mechanically stable and confirm the design retained for the series production of the $\mathbf{4 0 0}$ LHC main quadrupoles.
\end{abstract}

Index Terms-Capacitive gauges, magnetic measurements, quadrupole, quench antennas, training.

\section{INTRODUCTION}

FTER the successful construction and testing of the two first prototypes Q1 and Q2 in 1994, the construction of three new-design main quadrupole magnets for the arc short straight sections of the Large Hadron Collider has been performed by CEA/Saclay in collaboration with CERN [1]. These $3.25 \mathrm{~m}$ long twin aperture quadrupoles are included in a cold mass with two corrector magnets. The two first newly designed cold masses, SSS3 and SSS4, have already been tested at CERN [2]. This paper describes the results of the cryogenics tests of the third one, SSS5, at CEA/Saclay before being delivered to CERN. Contrary to SSS3 and SSS4, capacitive gauges were installed in the pole parts to follow the azimuthal coil stresses from the construction to the powering. Training behavior and quench localization by means of quench antenna are also discussed as proof of the mechanical stability of SSS5.

Manuscript received September 24, 2001.

F. Simon, C. Gourdin, T. Schild, J. Deregel, A. Devred, B. Hervieu, M. Peyrot and J. M. Rifflet are with CEA Saclay, France.

T. Tortschanoff is with CERN, Geneva, Switzerland.

T. Ogitsu and K. Tsuchiya are with KEK, Tsukuba, Japan.

Publisher Item Identifier S 1051-8223(02)03473-5.

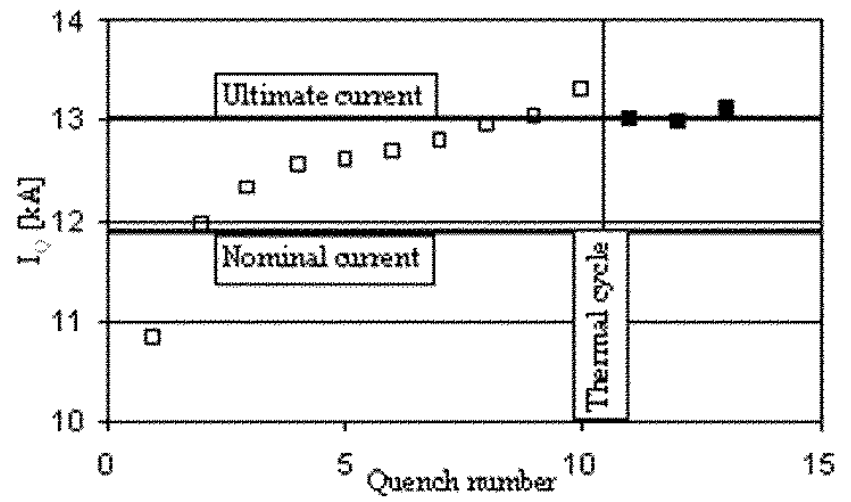

Fig. 1. Training curve of SSS5.

\section{ELECTRICAL TESTS}

\section{A. Training}

The training of the SSS5 (see Fig. 1) quadrupole magnet was short since there was only one quench registered below the nominal operating value of $11870 \mathrm{~A}$ (i.e., $223 \mathrm{~T} / \mathrm{m}$ ). After eight quenches, the magnet has been powered up to $12825 \mathrm{~A}$ (i.e., $242 \mathrm{~T} / \mathrm{m}$ ) which was set as the upper excitation limit. After a thermal cycle, the magnet did not experience quenches below the nominal operational value and went above the ultimate current.

Before the 12th quench, SSS5 was successfully powered up to the nominal current with ramp rates up to $408 \mathrm{~A} / \mathrm{s}$.

\section{B. Quench Analysis}

For the quench analysis, the magnet was equipped with voltage taps and quench antennas. Quench antennas were developed within the framework of a collaboration between KEK (Japan) and CEA. Three antennas made of two sextupole (normal coils $6 \mathrm{~N}$ and skew coils $6 \mathrm{~S}$ ) and two octupole ( $8 \mathrm{~N}$ and $8 \mathrm{~S})$ pick-up coils are inserted in each aperture of the magnet. There is one antenna at each end [the so-called Feed-End (FE) and Non Feed-End (NFE)], and one in the middle of the straight part (MID). The principle is to detect the displacement of a current line due to the quench. Thanks to voltage measurements (see Fig. 2), it is then possible to calculate the position and the velocity of the current line within the cross-section of the magnet. These are the vector displacements drawn in the magnet cross-section of Fig. 2. Detailed calculations have already been published by T. Ogitsu [3].

The maximum MIIT's value measured is about 31 MIITs. According to an adiabatic calculation it means that the maximum 


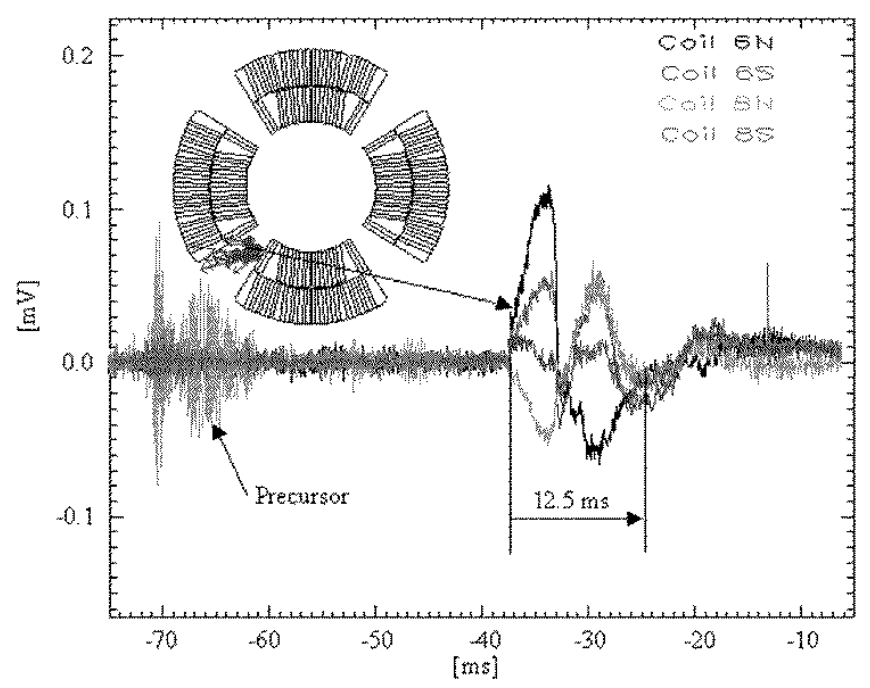

Fig. 2. Quench antenna analysis: these are the voltage drop measured on the four pick-up coils of a quench antenna during Quench number one. The inset shows the resulting quench localization.

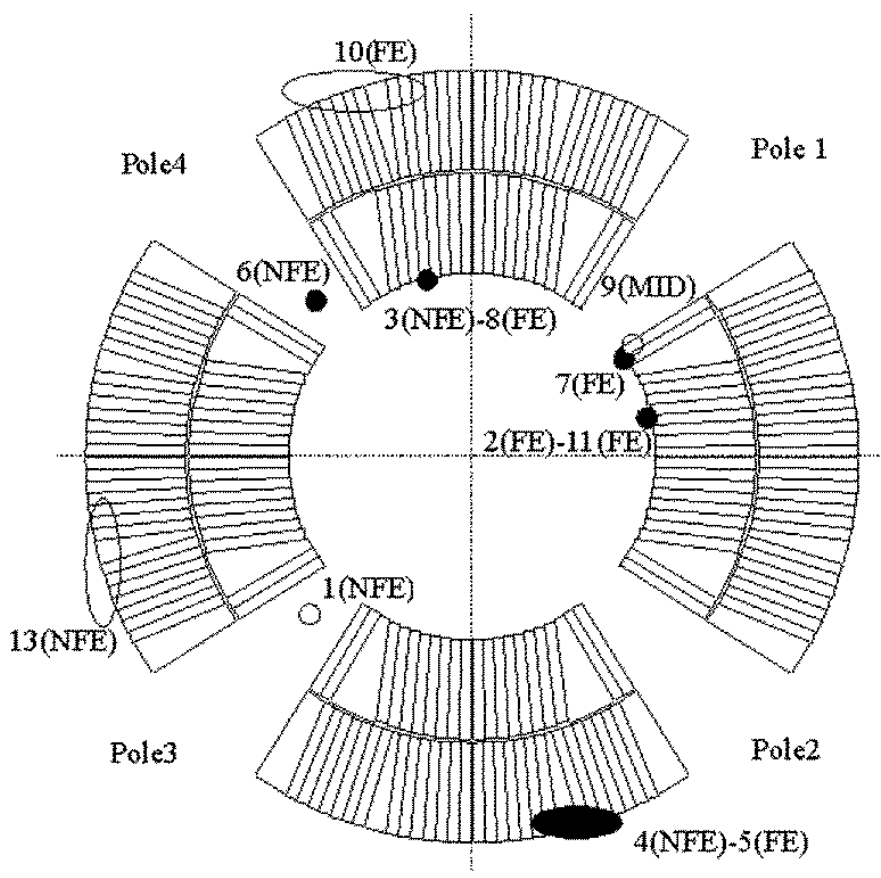

Fig. 3. Quench localization: - Quench in aperture one, $\bigcirc-$ Quench in aperture two. (FE) means a quench in Feed-End, (NFE) quench in Non Feed-End, and (MID) in the middle of the straight part.

temperature of the magnet during a quench, even at $13000 \mathrm{~A}$, is below $150 \mathrm{~K}$. This value is above the design maximum temperature, i.e., $100 \mathrm{~K}$, but still acceptable.

Fig. 2 presents a typical antenna voltage measurements for quench number 1 . First, an oscillating signal on the $8 \mathrm{~N}$ pick-up coil can be observed. This oscillation could be related to a vibration of the magnet conductor [4]. Such oscillations have been observed up to $90 \mathrm{~s}$ before the quench, and only on the octupole pick-up coils. The frequency of the vibration is above the maximum measurable frequency, $5 \mathrm{kHz}$.

Using these analysis data, it is also possible to determine the quench propagation velocity by measuring the time needed to go through a pick-up. For example, for quench number 1, a time

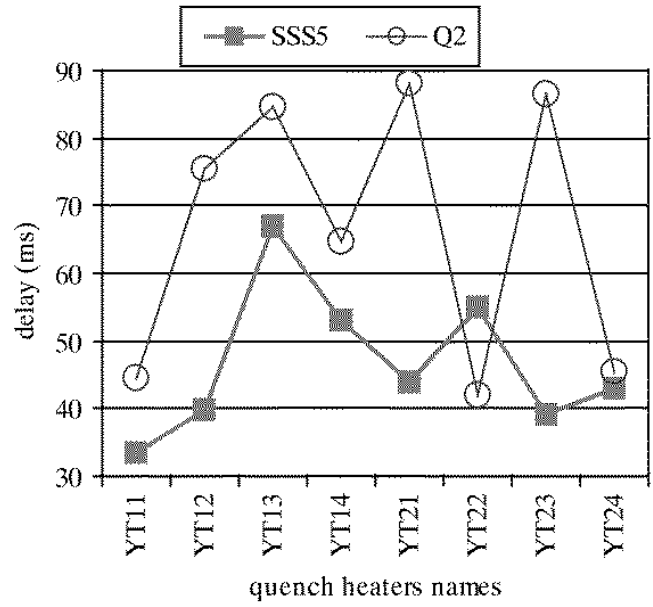

Fig. 4. Delay for quenches at $10 \mathrm{kA}$ provoked by a single quench heater. Q2 is a previous design quadrupole and SSS5 is the new design.

of $12.5 \mathrm{~ms}$ is measured (see Fig. 2). Given a pick-up length of $350 \mathrm{~mm}$, the quench velocity is then about $12 \mathrm{~m} / \mathrm{s}$. It is also possible to measure the time needed to reach the next antenna. In that case, $11 \mathrm{~m} / \mathrm{s}$ have been found. The quench propagation velocity measured in that way ranges between $11 \mathrm{~m} / \mathrm{s}$ (Quench 1), and $22 \mathrm{~m} / \mathrm{s}$ (Quench 2).

Fig. 3 presents the results of the quench antenna analyses for all quenches. The localization is quite accurate for the inner layer events, but gives only rough information for the outer layer events. Quench 1 and 6 are not located on a conductor because the quench occurs in a coil head. In that case the quench propagate symmetrically, and then the antenna locates the quench in between the most inner layers.

The weakest part of the magnet seems to be the feed end of pole one of aperture one as three quenches were ignited in that region.

\section{Quench Heaters}

For the newly designed prototype, the quench heaters were modified and made by a printed circuit technology. A U-shape stainless steel strip is deposed on a $125 \mu \mathrm{m}$-thick polyimide foil and covered by another $50 \mu \mathrm{m}$-thick polyimide foil. The thinner side is separated from the coils by a $75 \mu \mathrm{m}$-foil. A quench heater covers 2 coils and there are 4 quench heaters par aperture. For the prototypes, all the 8 quench heaters can be powered individually.

Fig. 4 shows the delay for each heater to quench the magnet while the current in the quadrupole is $10 \mathrm{kA}$. The delay is the time between the instant when the quench heater is individually triggered and when the voltage of the pole goes over $20 \mathrm{mV}$. It can be observed that the new quench heaters (SSS5 curve) trigger the magnet faster than the old ones, measured in Q2 (47 ms on average compared to $67 \mathrm{~ms}$ ). They appear also more reproducible since the standard deviation of Q2 is twice the one of SSS5.

The variations in SSS5 cannot be explained by the resistance of the quench heater or the discharge bench. They are likely due to differences in thermal contacts between the quench heater and the coil. 


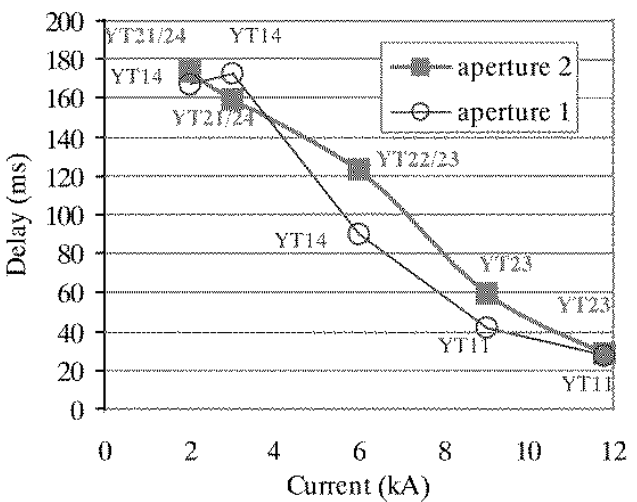

Fig. 5. Delay to quench SSS5 at different current when the four quench heaters of an aperture are triggered simultaneously.

A second test consists in measuring the quench delay when all the heaters of one aperture are triggered simultaneously for different currents (Fig. 5). First of all, the current under which the heaters can not trigger the magnet is between 1000 and $2000 \mathrm{~A}$. Secondly, it can be noted that the most efficient quench heater of each aperture in the previous tests (YT11 and YT23) is obviously the one that triggers SSS5 in this test. But this is true only at high current. At lower currents, the voltage taps show that the quenches start in coils that are not covered by YT11 or YT23. Studies are undertaken to explain this different mode of triggering.

For the final magnets four quench heaters will be connected in series. Each set includes two opposite quench heaters of both apertures. In this way, each set is in contact with all the coils of the twin-aperture magnet and the protection is redundant. It has been checked that the magnet can be quenched at $2 \mathrm{kA}$ with only two opposite quench heaters of an aperture (the less efficient quench heaters YT22 and YT24 were used).

All these electrical tests show that the new-design prototypes keep the quench memory without any retraining effect after a thermal cycle and have better quench heaters. Only two training quenches occurred in the internal turn of the coil where the magnetic field is maximum and none in the conductor ramp between the first to the second layer.

\section{MechanicAl Tests}

\section{A. Description}

Capacitive force sensors are incorporated to monitor the history of the compressive stress in the LHC arc quadrupole magnet throughout assembly, cool-down and energization. The sensors are designed as printed circuits, consisting of an alternated stack of dielectric and electrodes. The dielectric is a polyimide film without adhesives, and the electrodes are made of copper. The sensors exhibit near linear capacitance-force curves, a low hysteresis and a good accuracy (less than $3 \mathrm{MPa}$ ). The sensors are insensitive to magnetic field [5].

\section{B. Results}

The value of the pre-stress in the coil assembly depends of the successive steps of loading history, from the collaring to cool down and to excitation.
The collaring process is performed in three stages. The first stage consists of pressing the side frame to push radially the collar. The compressive stress is low. The second is the insertion of the intermediate keys; the value of pre-stress is about $55 \mathrm{MPa}$ when the step was completed. The last stage is pressing on the final keys to apply the final pre-stress on the coils by the insertion of the final keys. The final pre-stress is $100 \mathrm{MPa}$, with no overstress during the collaring process.

During the cool down, from $300 \mathrm{~K}$ to $4.2 \mathrm{~K}$, the loss of compressive stress is due to the thermal differential shrinkage. So the final stress in the coil assembly after cool-down is $78 \mathrm{MPa}$.

The last stage corresponds to the energization of the magnet. The current is increased until a quench which occurs at $12825 \mathrm{~A}$. The compressive stress at this point is still $53 \mathrm{MPa}$. So, the coil are always under compressive stress. This value is in good agreement with predictions from numerical analysis [6].

\section{Magnetic Measurements}

During the construction of the 3 prototypes, magnetic measurements at room temperature were performed at CEA/Saclay with a CERN measuring system [7]. Tests were done after collaring of the coils and after the assembly of two collared coils in the cold mass [8]. A strong correlation was found between these two stages [2]. SSS3 and SSS4 have also undergone cold magnetic measurements at CERN with a similar but different device. Eddy and persistent current effects were studied and the geometric harmonics were found to be quite similar to the warm ones.

Before being delivered to CERN, SSS5 was tested at cryogenic temperature at CEA with Saclay's device [9]. While this device is based on the rotating coil array technique like CERN's system, it differs on the compensation method. The compensation consists in combining the signals from the coil array in order to buck-out the dipole and quadrupole field and only conserve the higher harmonics. In CEA's system, three radial and the two tangential coils all with electronically adjustable gains allow to bring the dipole bucking ratio to 1000 while the quadrupole bucking is 2000 .

Two campaigns of loadline measurements (multipoles as a function of current) were performed, before and after the thermal cycle. The measurements were taken during the successive plateaus of a staircase-type current loop with a $50 \mathrm{~A} / \mathrm{s}-$ ramp rate after a standardization cycle of up to $11870 \mathrm{~A}$. Unfortunately there is no measurement in the virgin state of the magnet because the first quench occurs under $11870 \mathrm{~A}$. Thus, the magnet coils may have moved between the warm and cold tests.

Table I compares the integrated multipoles between the warm and cold measurements. Except for the sextupole $c_{3}$ component in the aperture 1 and the octupole $c_{4}$ in the aperture 2, the results are quite similar between the warm and cold measurements. As the system was incorrectly positioned on the connection side, the sextupole differs in fact by about 1 unit. Iron saturation effect cannot explain these discrepancies. Coil displacements or the difference between the two measuring systems are being studied presently. 
TABLE I

SSS5 INTEGRATED MULTIPOLES

\begin{tabular}{cccc}
\hline Multipole modulus & Warm & Cold 1 & Cold 2 \\
\hline & SSS5 aperture 1 \\
\hline$c_{3}$ & 3.24 & 1.85 & 1.80 \\
\hline$c_{4}$ & 0.98 & 0.83 & 0.75 \\
\hline$c_{5}$ & 0.67 & 0.64 & 0.56 \\
\hline$c_{6}$ & 6.03 & 6.22 & 6.34 \\
\hline$c_{10}$ & 0.20 & 0.21 & 0.27 \\
\hline \multicolumn{4}{c}{ SSS5 aperture 2 } \\
\hline$c_{3}$ & 1.88 & 1.37 & 1.26 \\
\hline$c_{4}$ & 3.00 & 4.17 & 4.18 \\
\hline$c_{5}$ & 0.26 & 0.09 & 0.19 \\
\hline$c_{6}$ & 5.79 & 6.09 & 6.18 \\
\hline$c_{10}$ & 0.20 & 0.19 & 0.21 \\
\hline
\end{tabular}

These cold tests will be repeated at CERN in the same conditions as for SSS3 and SSS4 in order to have more consistent statistical data. Nevertheless, a preliminary statistics shows a good correlation between warm and cold measurements. Considering the three cold masses, the mean values differ by a maximum of 0.07 units for the allowed multipoles $\left(b_{6}, a_{6}, b_{10}, \ldots\right)$ and 0.2 for the others (excepted $a_{3}$ with 0.4 unit).

On the other hand, there is no variation between the two cold campaigns. This confirms that there is no magnet deformation after a thermal cycle.

In order to estimate the influence of persistent magnetization currents during the transfer function tests, the ramp rate was decreased to $2 \mathrm{~A} / \mathrm{s}$ when reaching the $730 \mathrm{~A}$-injection current. SSS5 values are close to those of the two other cold masses [10]. The mean value is close to what was recently computed $\left(-3.48\right.$ unit for the normal dodecapole $\left.b_{6}\right)$ but different from the initially estimated value [11] used for the conception of the magnets. On the other hand, the geometric $b_{6}$ was measured higher than expected. These both points will be corrected during the series fabrication by fitting the thickness of the protection sheet placed between the pole part of the collars and the coils.

\section{CONCLUSION}

The test results of SSS5 are in accordance with our expectations and similar to those of SSS3 and SSS4. This proves that the magnet quality is reproducible over the manufacturing. The main magnetic properties at $1.9 \mathrm{~K}$ can be predicted with good accuracy by magnetic measurements at room temperature of the collared coils. This important point allows to reject an imperfect magnet at the beginning of the fabrication. Finally the mechanical stability is now known to be insensitive to thermal cycle. All these experimental results make us confident for the series-fabrication and the life-time of the main LHC quadrupole magnets [12].

\section{REFERENCES}

[1] M. Peyrot, J. M. Rifflet, F. Simon, P. Vedrine, and T. Tortschanoff, "Construction of the new prototype of main quadrupole cold masses for the arc short straight section of LHC," in Proc. MT-16, Ponte Vedra Beach, FL, USA, 1999.

[2] T. Tortschanoff, J. Billan, L. Bottura, A. Siemko, F. Simon, and M. Peyrot et al., "Performance of series-design prototype main quadrupoles for the LHC," in Proc. EPAC-2000, Vienna, Austria, 2000.

[3] T. Ogitsu et al., "Quench antenna for superconducting particle accelerator magnets," IEEE Trans. A.S.C., vol. 3, no. 1, p. 781, 1993.

[4] B. Dezillie, K. Artoos, A. Siemko, R. Mompo, and D. Tommasini, "Study of mechanical disturbances in superconducting magnets using piezoelectric sensors and quench antenna,", unpublished.

[5] L. Viellard, C. Gourdin, P. Vedrine, and B. Gallet, "Capacitive force sensors for prestress in accelerator magnets coils," in Proc. CEC/ICMC 1999, Québec, Canada, 1999.

[6] C. Gourdin, L. Champaney, and P. Vedrine, "The use of a new modular approach in the mechanical design of superconducting magnets," in Proc. MT-17, Geneva, Switzerland, submitted for publication.

[7] J. Billan et al., "Ambient temperature field measuring system for LHC superconducting dipoles," in Proc MT-14, Tampere, Finland, 1995.

[8] E. Todesco, S. Redaelli, V. Remondino, W. Scandale, F. Simon, and J. M. Rifflet et al., "Analysis of warm magnetic measurements of the first series-design prototypes of the LHC main quadrupoles," in Proc. EPAC 2000, Vienna, Austria, 2000.

[9] A. Devred, J. Belorgey, J. M. Rifflet, P. Vedrine, J. Deregel, and J. Billan et al., "Field quality of LHC/Saclay arc quadrupole magnet prototype," in Proc. EPAC 1996, Sitges, Spain, 1996, pp. 2225-2227.

[10] T. Torschanoff, J. Billan, V. Remondino, A. Siemko, N. Smirnov, and M. Peyrot et al., "Performance of prototypes and start up of series fabrication of the LHC arc quadrupoles," in Proc. PAC 2001, Chicago, USA, 2001.

[11] R. Wolf and S. Le Naour, "The expected persistent current field errors in the LHC main dipole and quadrupole," LHC Project Note 230, unpublished.

[12] R. Burgmer, R. Bussjäger, D. Krischel, J. M. Rifflet, P. Schmidt, and T. Tortschanoff et al., "Launching of series fabrication of the LHC main quadrupoles," in Proc. MT-17, Geneva, Switzerland, submitted for publication. 